\title{
Imaging Dural Sinus Thrombosis
}

The acute onset of irreversible neurological deficit is referred to as a "stroke" and is the third leading cause of death and the major cause of adult long-term disability in the United States. (Fischbein et al., 2000). Obstruction of the venous sinuses by thrombosis accounts for $\sim 1 \%$ of strokes. Thrombosis of the superior saggital sinus is most frequent, with involvement of transverse, sigmoid, and cavernous sinuses less often. Venous thrombosis may involve the dural sinuses, the deep venous system, and the superficial cortical veins separately or in any combination.

Patients presenting with seizures more often have thrombosis of the superficial cortical veins. These patients have a worse prognosis than those with isolated dural sinus thrombosis. Early diagnosis and treatment significantly improve prognosis. Ideally, therapy can be started before the patient has developed significant edema and/or hemorrhage. Mortality figures range from $20 \%$ to $75 \%$ with a trend of lowered rates with increased availability of MRI.

In $\sim 40 \%$ of cases of dural sinus thrombosis, there is retrograde clot propagation into the cortical or deep medullary veins. While the causes of venous thrombosis can be divided into septic and aseptic etiologies, $25 \%$ remain unknown, being classified as idiopathic (Fischbein et al., 2000).

\section{IMAGING DURAL SINUSES BY TIME-OF-FLIGHT MRV}

Magnetic resonance imaging with magnetic resonance venography (MRV) is the technique of choice in the diagnosis and follow-up of cerebral venous thrombosis. Thrombosis may be identified on spin echo images as an absence of the normal flow void. On MRV, thrombosis manifests itself as lack of signal. Thrombus signal intensity differs on $T_{1}$ - and $T_{2}$-weighted spin echo images and evolves with the phases of hemoglobin degradation. Recognition of artifacts and pitfalls related to MRI techniques is crucial to correctly interpret the presence or absence of venous sinus thrombosis.

The following sequences comprise the authors' preferred protocol for evaluating dural sinus thrombosis, (1) three-plane positioning scout; (2) time-of-flight MRV; (3) noncontrast spin echo sagittal $T_{1}$-weighted; (4) non-contrast coronal turbo spin echo proton density $/ T_{2}$-weighted; and (5) spin echo coronal with flow compensation. These imaging sequences take $\sim 16 \mathrm{~min}$ and $3 \mathrm{sec}$ of imaging time in addition to set up time between sequences.

Table A2.1.1 lists the equipment needed to perform the following imaging sequences. The available gradient strength will depend on the scanner, and the echo times given below will be modified accordingly (i.e., the smaller the gradient strength, the longer the echo

Table A2.1.1 Equipment Specifications Needed to Perform Imaging Sequences

\begin{tabular}{ll}
\hline Coil type & Circularly polarized head coil \\
Field strength & $1.5 \mathrm{~T}$ \\
Gradient strength & Minimum of $15 \mathrm{mT} / \mathrm{m}$ \\
Positioning cushion or head & Yes \\
stabilizers & \\
Knee cushion & Yes \\
Use of contrast agents & No \\
Pulse oximeter & If patient requires sedation \\
\hline
\end{tabular}

Contributed by F. Allan Midyett, Suresh Mukherji, and Laurie Fisher

Current Protocols in Magnetic Resonance Imaging (2001) A2.1.1-A2.1.12

Copyright @ 2001 by John Wiley \& Sons, Inc.
BASIC

PROTOCOL

Cerebral Venous

Lesions

A2.1.1 
time for a particular scan). The authors use a Siemens Vision $1.5 \mathrm{~T}$ system, but they expect to run the same sequences in this unit on other machines made by other MR manufacturers.

NOTE: Be sure that technologists and nurses have immediate access to any emergency equipment that may be relevant to a given study, or that may be needed for a particular patient, such as crash carts or oxygen.

\section{Set up patient and equipment}

1. Interview the patient to assess for contraindications such as cardiac pacemaker, implanted mechanical devices, and/or ferromagnetic materials. Also, determine if the patient will need sedation medication, which will necessitate the need for appropriate monitoring equipment.

Each patient or legal guardian, prior to bringing the patient into the exam area, must sign a screening form.

The presence of ferromagnetic materials may be a health hazard to the patient while in the magnetic field and/or adversely affect image quality. To determine the safety of scanning such ferromagnetic materials, see Shellock (1996).

The presence of ferromagnetic materials in the globe of the eye is contraindicated for MRI. Patients with prior metal exposure to the eye should have plain X-rays of the orbital area to ensure that all metal has been removed prior to placing them in the magnetic field (Shellock, 1996).

2. If the procedure is a research protocol, have the patient sign any necessary consent form.

3. Request the patient to change into a gown and remove all personal effects such as, jewelry, hearing aids, glasses, etc., prior to entering the MRI scan room. All personal belongings should be secured during the examination.

4. Have the patient wash off any mascara and other makeup to avoid local tissue heating and artifacts.

5. Explain the procedure to the patient and record relevant clinical history. Ensure that the patient understands what is expected and ask if he or she has questions. Answer appropriately.

6. Set up the exam room by securing the circularly polarized (CP)-head coil onto the table and providing a clean exam table.

7. Escort the patient to the MR examination room and position him or her according to the exam to be performed. Review the following items with the patient:

a. Provide earplugs or headphones to the patient to minimize the gradient noise but assure the patient that the patient should still be able to hear you.

b. Provide the patient with a safety squeeze-bulb and demonstrate how it works. Explain to the patient that he or she may use the squeeze-bulb if assistance is needed during the exam.

c. Explain to the patient that you will be talking to him or her between imaging sequences that will be when the loud knocking noise stops.

d. Explain to the patient that it is imperative that he or she remains motionless during the loud knocking noise to ensure good results. Also, explain that they should not reposition their body between imaging sequences.

Imaging Dural Sinus Thrombosis

A2.1.2

e. The patient may call out at any time if he or she feels it necessary. 
f. Provide the patient with an estimate of the examination length. Although the total scan time is 16 min and $3 \mathrm{sec}$, because of set-up time between sequences, tell the patient that this exam will take $\sim 20$ min.

8. Position a support under the patient's knees to enhance comfort.

9. If the patient is unable to hold still, provide appropriate sedation.

10. Secure the patient's head with positioning sponges or head stabilizers.

11. Center the patient's nasion to the coil using the laser light.

12. Advance the patient couch to isocenter.

\section{Sequence 1: Rapid three-plane positioning scout}

13. To validate the patient's position and to have a reference to prescribe successive imaging sequences, acquire a three-plane orthogonal scout sequence. See Table A2.1.2 for specific parameters. Run the scan.

Most MR scanners can be programmed to acquire the scout automatically after coil tuning or after the patient has been placed in isocenter (for systems that do not require tuning). Siemens acquires the scout in three planes by selecting "various."

\section{Sequence 2: Time-of-flight MRV-sagittal sinus}

14. Display both the sagittal and transverse scout images in two separate quadrants on the scan monitor.

15. Change imaging parameters to those listed in Table A2.1.3. Position slices to midline of the transverse scout with a sagittal to coronal angle of approximately $-12^{\circ}$ (this will reduce in-plane saturation effects). Position the presaturation band off the sagittal scout image angled in a manner to saturate the facial sinuses (be sure not to "clip" any of the sagittal sinus).

16. Instruct the patient to remain motionless, as the scan will begin and last for $\sim 9 \mathrm{~min}$. Run a time-of-flight magnetic resonance venogram (MRV) of the superior sagittal sinus according to the parameters in Table A2.1.3.

This sequence is most important in demonstrating flow within the dural sinuses as well as potentially showing the location and cause of obstruction including thrombi.

\section{Sequence 3: Noncontrast spin echo sagittal $T_{1}$-weighted sequence}

17. Some patients have this examination because of findings noted on a routine cranial MR. If not already provided as a part of a recent study, run a noncontrast spin echo sagittal $T_{1}$-weighed scan using the parameters in Table A2.1.4.

\section{Sequence 4: Noncontrast coronal multiecho spin echo}

18. Run a noncontrast coronal multiecho scan according to Table A2.1.5. This is a dual echo turbo spin echo sequence that acquires both proton density and $T_{2}$-weighted images simultaneously.

In this study, look for normal flow voids in the venous sinuses. These flow voids may be compromised by signal from blood products on $T_{2}$-weighted images. There tend to be fewer of these artifacts on the proton density weighted images. The proton density weighted images come as a bonus with the $T_{2}$-weighted images without any additional expenditure of time and they are useful in this situation.

Sequence 5: Spin echo coronal with flow compensation

19. Run a spin echo coronal scan according to the parameters in Table A2.1.6.

Cerebral Venous

Lesions

A2.1.3 
Table A2.1.2 Imaging Parameters for Sequence 1: Scout Sequence

\begin{tabular}{ll}
\hline Patient position & $\begin{array}{l}\text { Supine } \\
\text { Scan type }\end{array}$ \\
Imaging plane (orientation) & $\begin{array}{l}\text { Transverse, sagittal, coronal } \\
\text { (various) }\end{array}$ \\
Central slice or volume center & Nasion \\
Echo time $\left(T_{\mathrm{E}}\right)$ & $6 \mathrm{msec}$ \\
Repeat time $\left(T_{\mathrm{R}}\right)$ & $15 \mathrm{msec}$ \\
Flip angle $(\mathrm{FA})$ & $30^{\circ}$ \\
Field of view $\left(\mathrm{FOV}_{\mathrm{x}}, \mathrm{FOV}_{\mathrm{y}}\right)$ & $300 \mathrm{~mm}, 300 \mathrm{~mm}$ \\
Resolution $(\Delta x, \Delta y)$ & $1.17 \mathrm{~mm}, 2.34 \mathrm{~mm}$ \\
Number of data points collected $\left(N_{\mathrm{x}}, N_{\mathrm{y}}\right)$ & 256,128 \\
Slice thickness $(\Delta z)$ & $8 \mathrm{~mm}$ \\
Number of slices & $3(\mathrm{one}$ per orientation) \\
Slice gap & Not applicable \\
Number of acquisitions $\left(N_{\mathrm{acq}}\right)$ & 1 \\
Swap read and phase encoding & No \\
Slice location & Not applicable \\
Saturation pulses & Not applicable \\
Slice series & Ascending caudal to cranial \\
Scan time & 7 sec \\
\hline
\end{tabular}

Table A2.1.3 Imaging Parameters for Sequence 2: Time-of-Flight MRV of the Sagittal Sinus

\begin{tabular}{|c|c|}
\hline Patient position & Supine \\
\hline Scan type & 2-D gradient echo \\
\hline Imaging plane (orientation) & Sagittal \\
\hline Central slice or volume center & $\begin{array}{l}\text { Slices centered to midline on } \\
\text { transverse scout }\end{array}$ \\
\hline Echo time $\left(T_{\mathrm{E}}\right)$ & $9 \mathrm{msec}$ \\
\hline Repeat time $\left(T_{\mathrm{R}}\right)$ & $30 \mathrm{msec}$ \\
\hline Flip angle (FA) & $50^{\circ}$ \\
\hline Field of view $\left(\mathrm{FOV}_{\mathrm{x}}, \mathrm{FOV}_{\mathrm{y}}\right)$ & $210 \mathrm{~mm}, 210 \mathrm{~mm}$ \\
\hline Resolution $(\Delta x, \Delta y)$ & $0.82 \mathrm{~mm}, 0.82 \mathrm{~mm}$ \\
\hline Number of data points collected $\left(N_{\mathrm{x}}, N_{\mathrm{y}}\right)$ & 256,256 \\
\hline Slice thickness $(\Delta z)$ & $3 \mathrm{~mm}$ \\
\hline Number of slices & 64 \\
\hline Slice gap & $1 \mathrm{~mm}$ \\
\hline Number of acquisitions $\left(N_{\mathrm{acq}}\right)$ & 1 \\
\hline Swap read and phase encoding & No \\
\hline Slice location & $\begin{array}{l}\text { Angle sagittal to coronal }-12^{\circ} \text { (to } \\
\text { reduce in-plane saturation) off } \\
\text { transverse scout image }\end{array}$ \\
\hline Saturation pulses & $\begin{array}{l}\text { Yes, one angled through facial } \\
\text { sinus area off sagittal scout image, } \\
60-\mathrm{mm} \text { thick }\end{array}$ \\
\hline Slice series & Ascending \\
\hline Scan time & $6 \mathrm{~min}, 43 \mathrm{sec}$ \\
\hline
\end{tabular}


Table A2.1.4 Imaging Parameters for Sequence 3: Spin Echo Sagittal $T_{1}$-Weighted Sequence

\begin{tabular}{|c|c|}
\hline Patient position & Supine \\
\hline Scan type & Spin echo \\
\hline Imaging plane (orientation) & Sagittal \\
\hline Central slice or volume center & $\begin{array}{l}\text { Slices centered to midline on } \\
\text { transverse scout }\end{array}$ \\
\hline Echo time $\left(T_{\mathrm{E}}\right)$ & $14 \mathrm{msec}$ \\
\hline Repeat time $\left(T_{\mathrm{R}}\right)$ & $510 \mathrm{msec}$ \\
\hline Flip angle (FA) & $90^{\circ}$ \\
\hline Field of view $\left(\mathrm{FOV}_{\mathrm{x}}, \mathrm{FOV}_{\mathrm{y}}\right)$ & $230 \mathrm{~mm}, 230 \mathrm{~mm}$ \\
\hline Resolution $(\Delta x, \Delta y)$ & $0.90 \mathrm{~mm}, 0.95 \mathrm{~mm}$ \\
\hline Number of data points collected $\left(N_{\mathrm{x}}, N_{\mathrm{y}}\right)$ & 256,243 \\
\hline Slice thickness $(\Delta z)$ & $6 \mathrm{~mm}$ \\
\hline Number of slices & 17 \\
\hline Slice gap & $1.8 \mathrm{~mm}$ \\
\hline Number of acquisitions $\left(N_{\text {acq }}\right)$ & 1 \\
\hline Swap read and phase encoding & No \\
\hline Slice location & $\begin{array}{l}\text { Equidistant between left and right } \\
\text { brain }\end{array}$ \\
\hline Saturation pulses & No \\
\hline Slice series & Interleaved \\
\hline Scan time & $2 \mathrm{~min}, 7 \mathrm{sec}$ \\
\hline
\end{tabular}

Table A2.1.5 Imaging Parameters for Sequence 4: Coronal Turbo Spin Echo Proton Density (PD)/ $T_{2}$-Weighted Images

\begin{tabular}{|c|c|}
\hline Patient position & Supine \\
\hline Scan type & Turbo spin echo (dual echo) \\
\hline Imaging plane (orientation) & Coronal \\
\hline Central slice or volume center & $\begin{array}{l}\text { Slices centered to midbrain on } \\
\text { sagittal scout }\end{array}$ \\
\hline Echo time $\left(T_{\mathrm{E}}\right)$ & $15 \mathrm{msec}$ and $105 \mathrm{msec}$ \\
\hline Echo train length (ETL) & 7 \\
\hline Repeat time $\left(T_{\mathrm{R}}\right)$ & $4500 \mathrm{msec}$ \\
\hline Flip angle (FA) & $180^{\circ a}$ \\
\hline Field of view $\left(\mathrm{FOV}_{\mathrm{x}}, \mathrm{FOV}_{\mathrm{y}}\right)$ & $\begin{array}{l}230 \mathrm{~mm}, 230 r \mathrm{~mm}, \text { with } r=3 / 4 \\
\text { (rectangular field of view) }\end{array}$ \\
\hline Resolution $(\Delta x, \Delta y)$ & $0.90 \mathrm{~mm}, 0.90 \mathrm{~mm}$ \\
\hline Number of data points collected $\left(N_{\mathrm{x}}, N_{\mathrm{y}}\right)$ & $\begin{array}{l}256,256 r, \text { with } r=3 / 4 \\
\text { (rectangular field of view) }\end{array}$ \\
\hline Slice thickness $(\Delta z)$ & $5 \mathrm{~mm}$ \\
\hline Number of slices & 24 \\
\hline Slice gap & $1.5 \mathrm{~mm}$ \\
\hline Number of acquisition $\left(N_{\mathrm{acq}}\right)$ & 1 \\
\hline Swap read and phase encoding & No \\
\hline Slice location & $\begin{array}{l}\text { Equidistant between anterior and } \\
\text { posterior brain }\end{array}$ \\
\hline Saturation pulses & No \\
\hline Slice series & Interleaved \\
\hline Scan time & $2 \mathrm{~min}, 3 \mathrm{sec}$ \\
\hline
\end{tabular}

Cerebral Venous

Lesions

A2.1.5 
Table A2.1.6 Imaging Parameters for Sequence 5: $T_{1}$-Weighted Spin Echo Coronal with Flow Compensation

\begin{tabular}{|c|c|}
\hline Patient position & Supine \\
\hline Scan type & Spin echo \\
\hline Imaging plane (orientation) & Coronal \\
\hline Central slice or volume center & $\begin{array}{l}\text { Slices centered to midbrain on } \\
\text { sagittal scout }\end{array}$ \\
\hline Echo time $\left(T_{\mathrm{E}}\right)$ & $17 \mathrm{msec}$ \\
\hline Repeat time $\left(T_{\mathrm{R}}\right)$ & $532 \mathrm{msec}$ \\
\hline Flip angle (FA) & $90^{\circ}$ \\
\hline Field of view $\left(\mathrm{FOV}_{\mathrm{x}}, \mathrm{FOV}_{\mathrm{y}}\right)$ & $\begin{array}{l}230 \mathrm{~mm}, 230 \mathrm{~mm} \text {, with } r=3 / 4 \\
\text { (rectangular field of view) }\end{array}$ \\
\hline Resolution $(\Delta x, \Delta y)$ & $0.90 \mathrm{~mm}, 0.90 \mathrm{~mm}$ \\
\hline Number of data points collected $\left(N_{\mathrm{x}}, N_{\mathrm{y}}\right)$ & $\begin{array}{l}256,256 r, \text { with } r=3 / 4 \\
\text { (rectangular field of view) }\end{array}$ \\
\hline Slice thickness $(\Delta z)$ & $5 \mathrm{~mm}$ \\
\hline Number of slices & 19 \\
\hline Slice gap & $1.5 \mathrm{~mm}$ \\
\hline Number of acquisitions $\left(N_{\mathrm{acq}}\right)$ & 2 \\
\hline Swap read and phase encoding & No \\
\hline Slice location & $\begin{array}{l}\text { Center midway anterior to } \\
\text { posterior brain }\end{array}$ \\
\hline Flow compensation & Yes \\
\hline Saturation pulses & No \\
\hline Slice series & Interleave \\
\hline Scan time & $3 \mathrm{~min} .26 \mathrm{sec}$ \\
\hline
\end{tabular}

Siemens calls this sequence gradient motion rephasing (GMR). It is very helpful in reducing flow related artifact that may hinder evaluation of dural sinuses, particularly in the posterior fossa. This sequence also accentuates the visibility of vessels, especially rapidly flowing blood. This is useful when the vein is narrow or there is a thin rim of flow around the periphery of a thrombus.

\section{COMMENTARY}

\section{Background Information}

Expectations have risen in the medical community to the point where clinicians expect to rule in or out dural thrombosis using noninvasive techniques. MR is the modality of choice in identifying and following treatment of dural thrombosis (Bianchi et al., 1998). MR may identify both the intravascular thrombus and the concomitant parenchymal complications. $\mathrm{Pa}$ tients may present with rapid or insidious onset of symptoms. Some patients present with rapid onset of altered consciousness and focal neurological deficits. Others present with gradual confusion, headache, papilledema, and cranial nerve palsies from increased intracranial pressure. Complications include cerebral edema, infarction, hemorrhage, hydrocephalus, and death. Predisposing factors are listed in the following table (Table A2.1.7; Cure and Van Tassel, 1994).

In involved veins, flow voids may be replaced by abnormal signal. The specific appearance of the abnormality varies according to the age of the thrombus and the imaging parameters used. The parenchymal findings vary with the stage of the process from normal to swelling with interstitial edema and subcortical hyperintensity on proton density weighted and $T_{2^{-}}$ weighted images, and in the more severe case will include the presence of hematoma (Tsai et al., 1995).
Imaging Dural Sinus Thrombosis 
Table A2.1.7 Factors Predisposing to Cerebral Venous Thrombosis

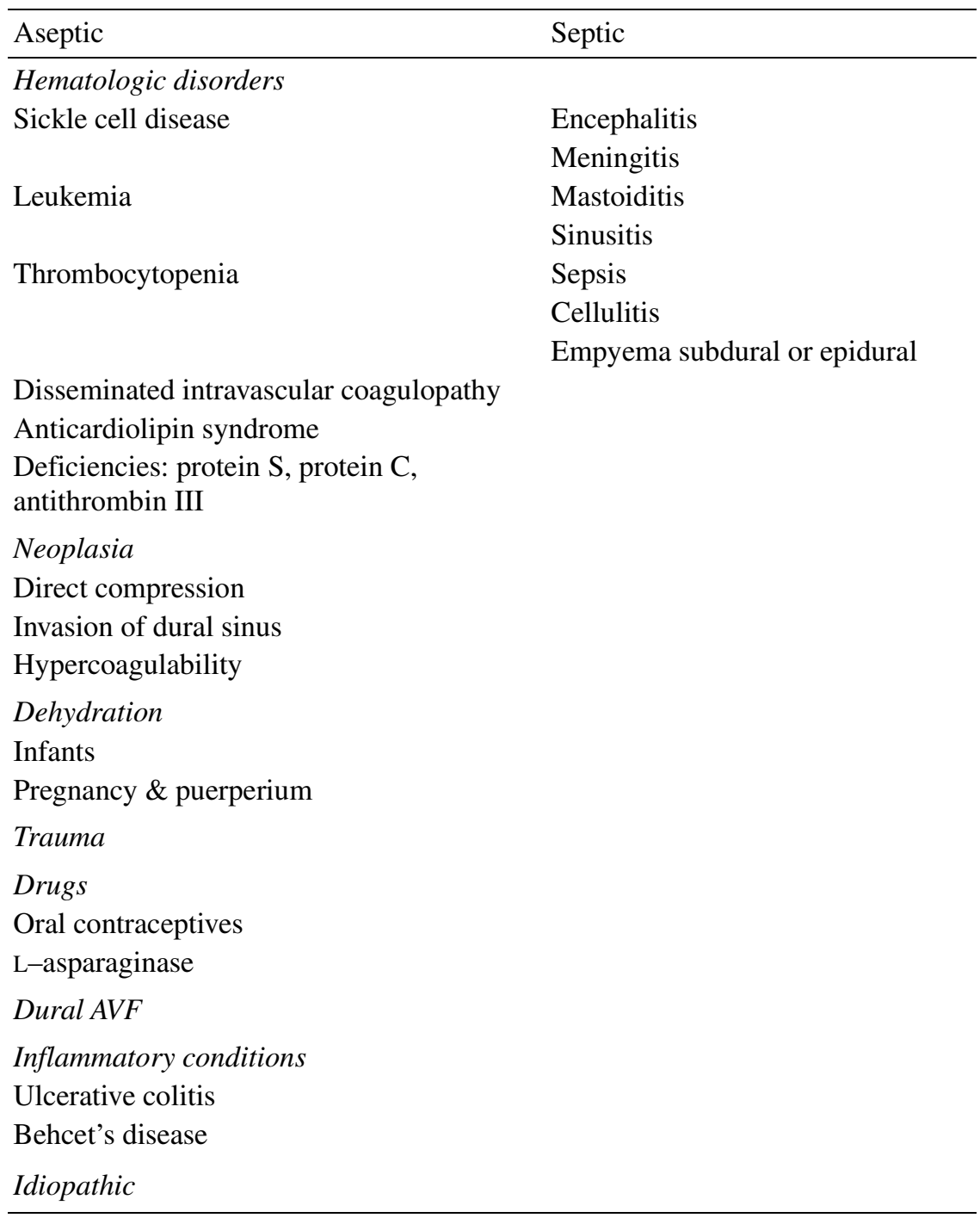

\section{Critical Parameters}

The authors routinely use time-of-flight for MRV. Occasionally, phase-contrast techniques are used if there is a question about direction of flow or if there is a problem with shinethrough from thrombus (for more information about phase contrast sequences, see the example given in UNIT Al.l, Table A1.1.8). The diagnosis of venous sinus thrombosis is supported by the absence of flow-related enhancement or motion-related phase shift. Because of its $T_{1}$ shortening, methemoglobin may exhibit shinethrough and mimic a patent sinus on time-offlight MRV. Findings must be correlated with those of $T_{1}$-weighted images. Phase-contrast MRV is a background subtraction technique and tends to eliminate signal from thrombus and hematomas. Phase-contrast MRV could be used when unable to resolve this problem with time-of-flight MRV and conventional $T_{1^{-}}$ weighted images (Yuh et al., 1994).

\section{Anticipated Results}

The goal in studying cerebral dural sinuses is to identify thrombosis and to allow the choice of timely treatment options. Interpretation of cerebral MRI examinations has potential for both false-positive and false-negative errors. Slow flow may simulate venous thrombosis on spin echo images creating a false positive finding. A false negative finding may be present with time-of-flight MRV in patients who have actual venous thrombosis. Here methemoglobin is a natural breakdown product present in clot. The $T_{1}$-shortening effects of methemoglobin can "shine-through" giving the illusion of
Cerebral Venous Lesions

A2.1.7 
a patent venous channel. The background subtraction technique of phase-contrast MRV may be helpful. This technique eliminates bright signal from stationary thrombus and hematomas (Fischbein et al., 2000). The following are some examples.

An 18-year-old male with sickle cell anemia has occlusion of his superior sagittal sinus with increased $T_{1}$ signal related to thrombus with methemoglobin formation (Fig. A2.1.1).

A 25-year-old pregnant female who has a hypercoagulable state presented with superior sagittal sinus thrombosis. There is total occlusion of most of her superior sagittal sinus with increased $T_{1}$ signal (Fig. A2.1.2). This is less intense than on the previous examination sug-

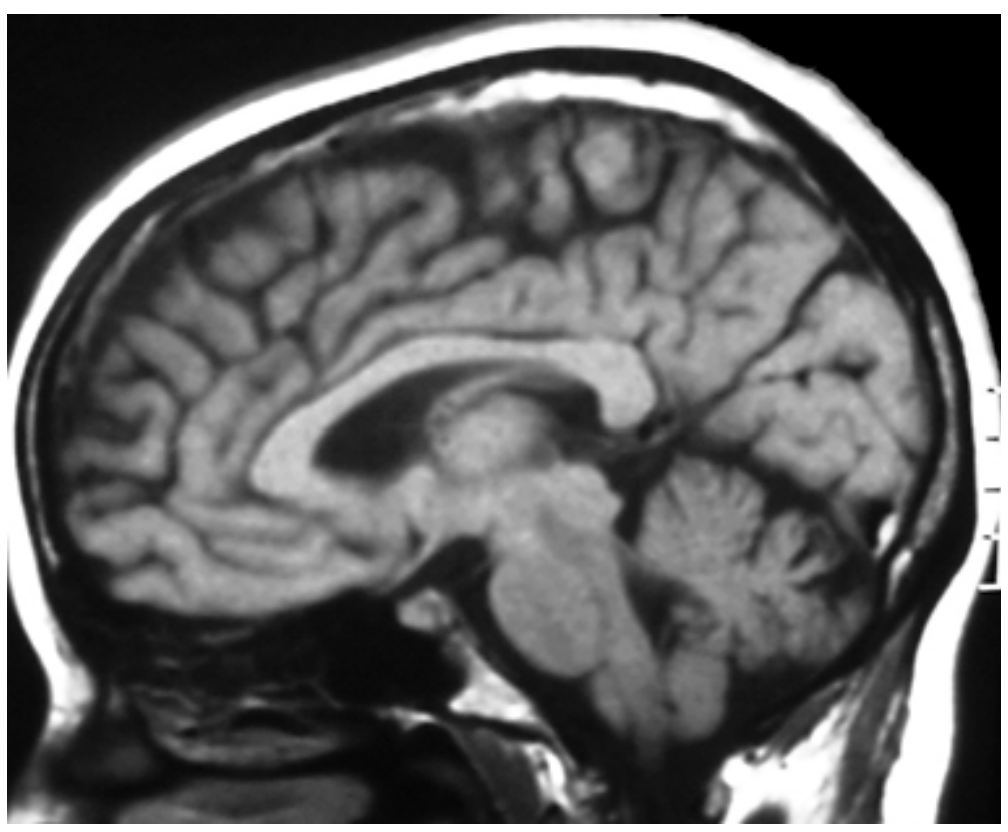

Figure A2.1.1 Superior sagittal thrombosis with bright $T_{1}$ signal from thrombus.

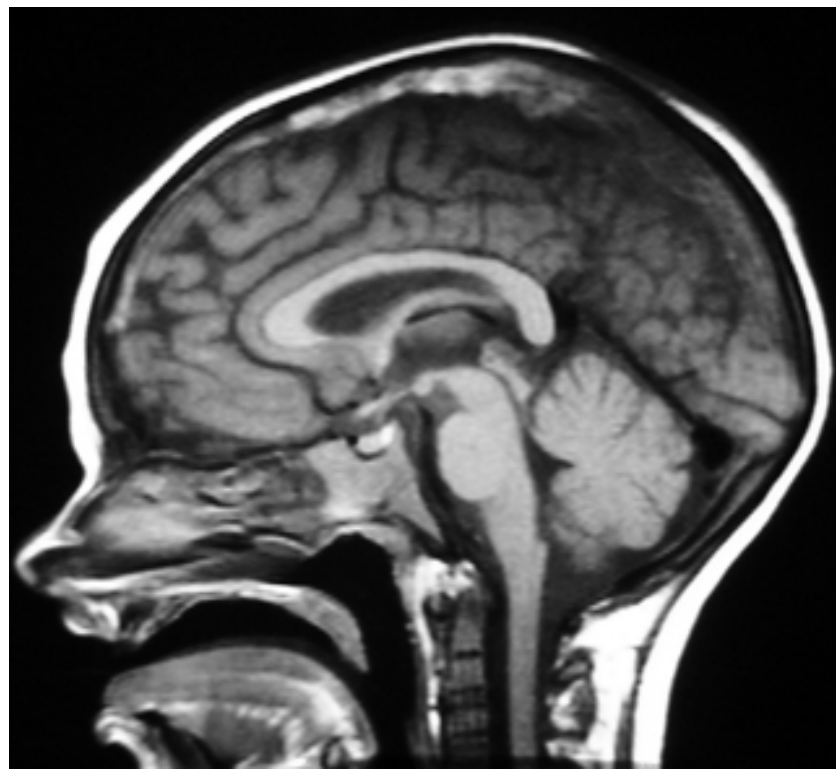

Figure A2.1.2 Superior sagittal sinus thrombosis with increased $T_{1}$ signal. This is less bright than the previous example. 
gesting that the clot is older than the one seen on the previous example (Fig. A2.1.1).

A 34-year-old man with leukemia has superior sagittal sinus thrombosis with clot that is almost isointense with brain (Fig. A2.1.3). The clot is older than the two previous examples. This is considerably less conspicuous than the two previous examples. With less signal throm- bus may be overlooked on conventional $T_{1^{-}}$ weighted images.

A 28-year-old female has occlusion of her transverse sinus with increased $T_{1}$ signal related to methemoglobin within the thrombus (Fig. A2.1.4). This much $T_{1}$ signal has the potential to cause "shine-through" artifact on MRV. Her only known risk factor was the use of oral

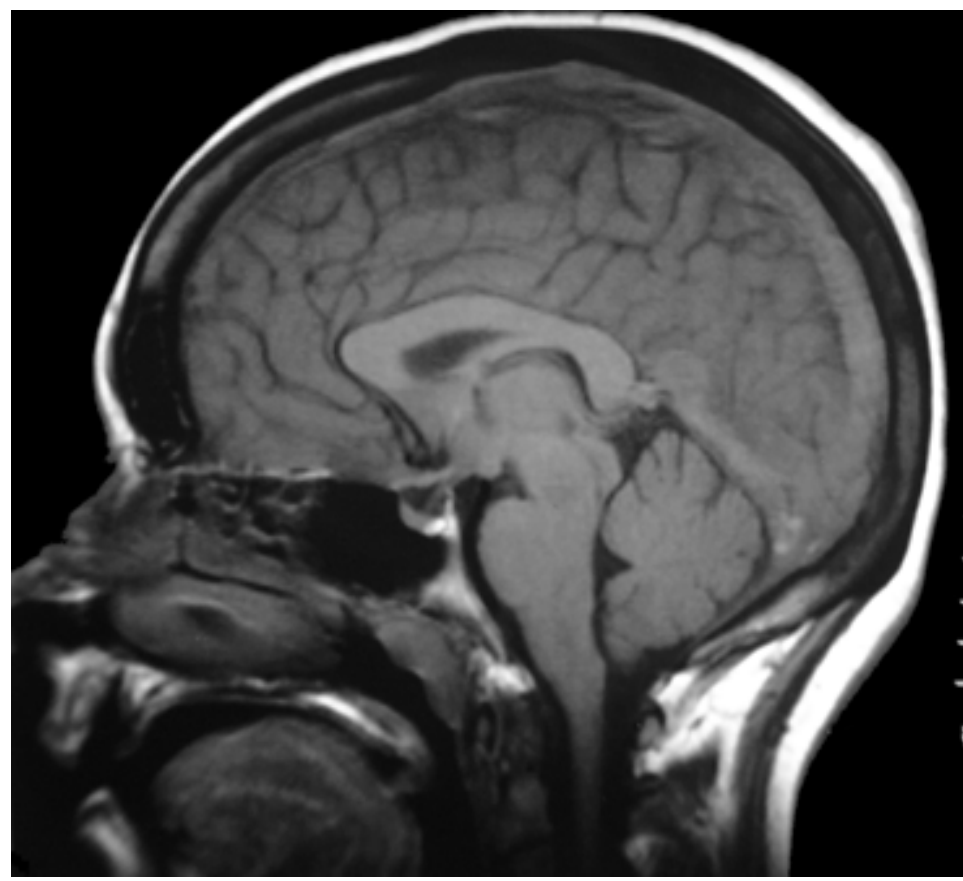

Figure A2.1.3 Superior sagittal sinus thrombosis with $T_{1}$ signal, which is almost isointense with brain.

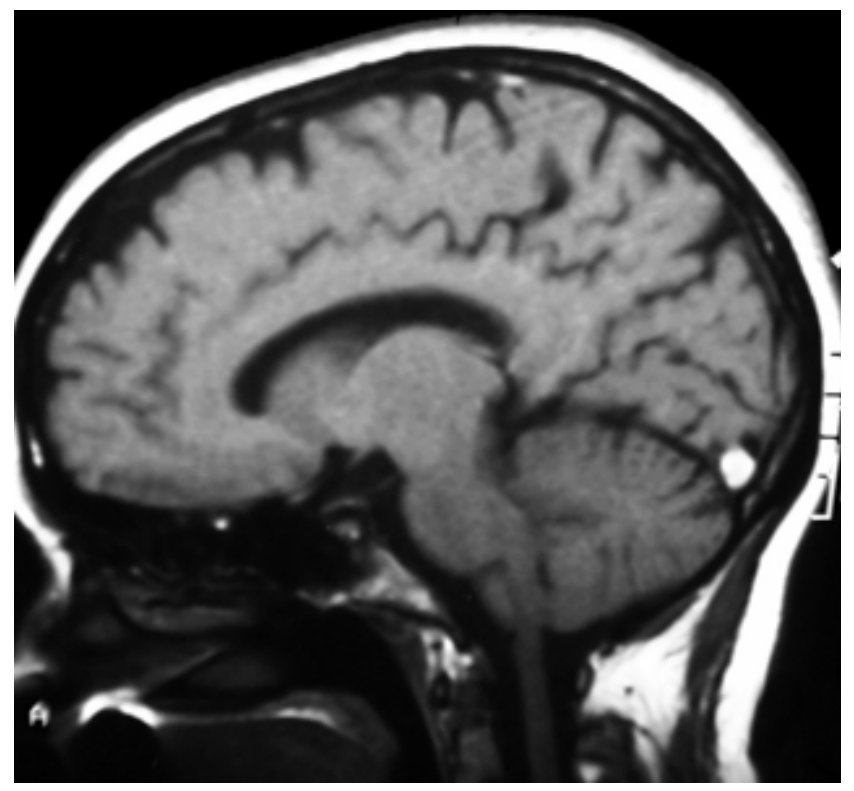

Figure A2.1.4 Occlusion of transverse sinus with high $T_{1}$ signal in clot. 
contraceptives and kept this case from being classified as idiopathic.

A 68-year-old male with thrombocytopenia has superior sinus thrombosis with partial blockage of the sinus (Fig. A2.1.5). Some flow is still demonstrated in the expected lumen of the sinus. The presence or absence of flow is better appreciated on MRV than on other sequences.

A 40-year-old female with disseminated intravascular coagulopathy has total occlusion of the superior sagittal sinus, the inferior sagittal sinus, the straight sinus and parts of the transverse sinuses (Fig. A2.1.6).

A 25-year-old male with mastoiditis had thrombosis of the right transverse sinus (Fig. A2.1.7 and Fig. A2.1.8). A conventional cranial MRI had demonstrated mastoiditis with increased $T_{2}$ signal in the region of mastoid air cells on the side of the thrombosis.

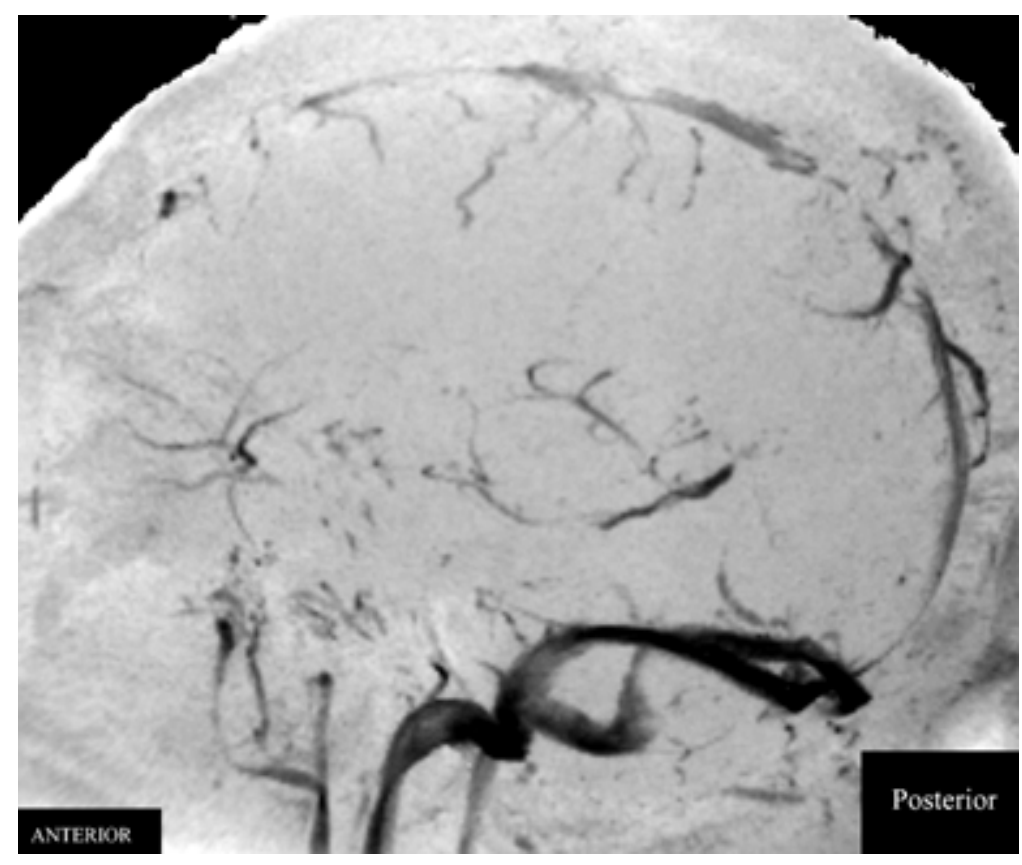

Figure A2.1.5 Superior sagittal sinus thrombosis with partial blockage on MRV.

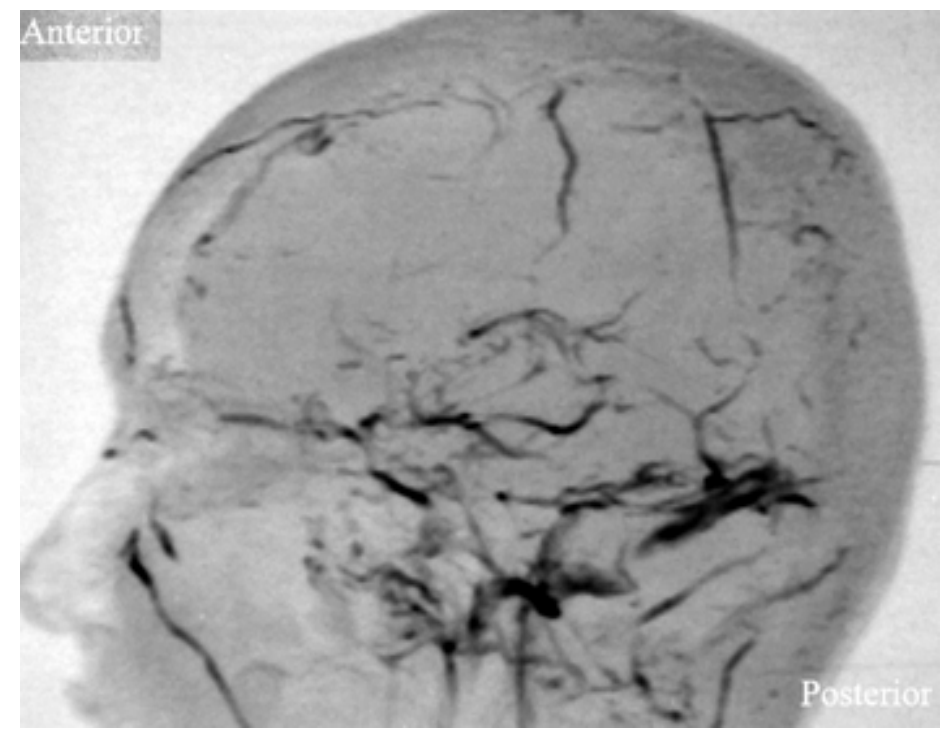

Figure A2.1.6 Superior sagittal sinus thrombosis with total occlusion on MRV. 


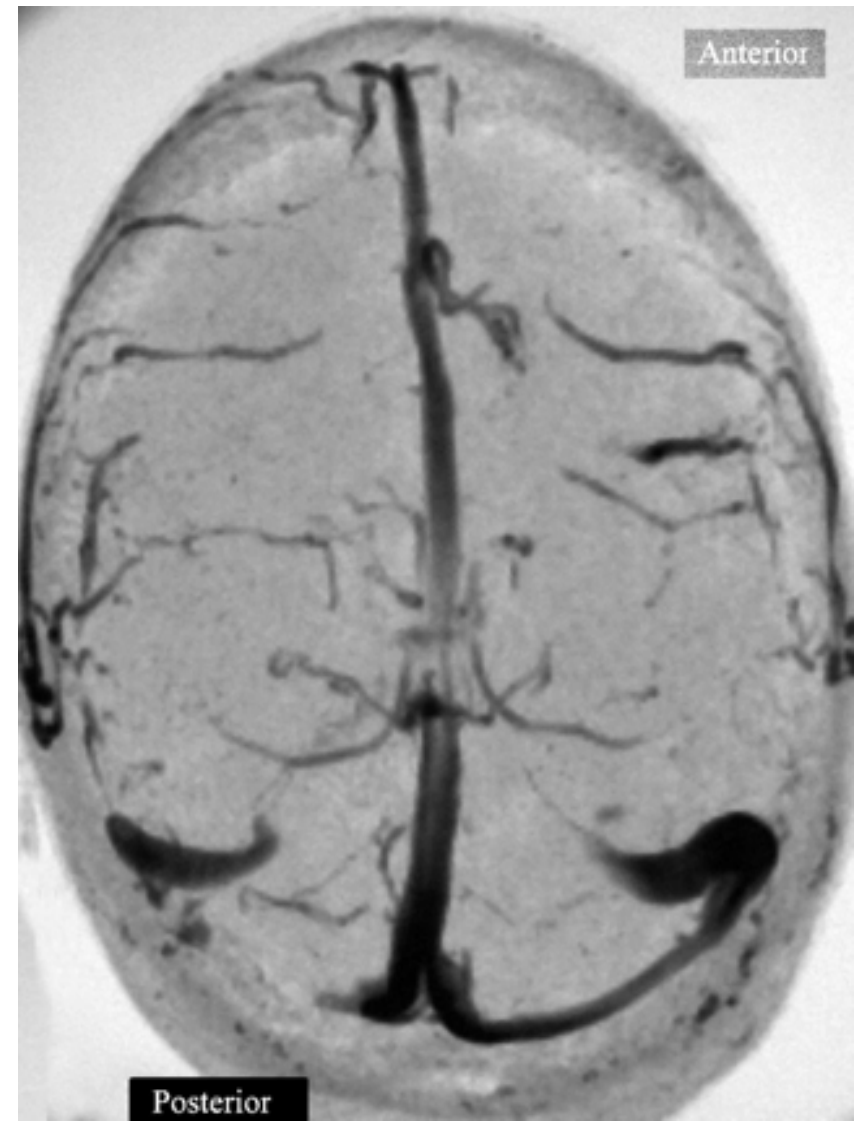

Figure A2.1.7 Occlusion transverse sinus on transverse MRV.

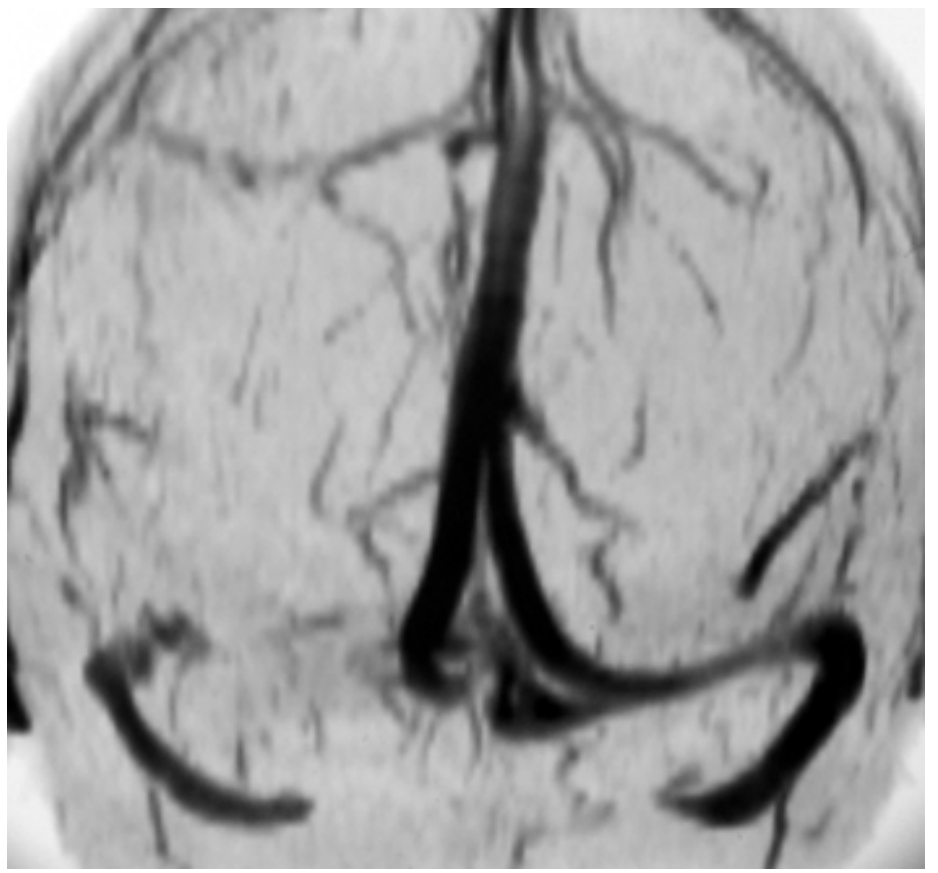

Figure A2.1.8 Occlusion transverse sinus on coronal MRV. 


\section{Literature Cited}

Bianchi, D., Maeder, P., Bogousslavsky, J., Schnyder, P., and Meuli, R. 1998. Diagnosis of cerebral venous thrombosis with routine magnetic resonance: An update. Eur. Neurol. 40:179-190.

Cure, J.K. and Van Tassel, P. 1994. Congenital and acquired abnormalities of the dural venous sinuses. Seminars US, CT, Magn. Reson. Imaging 15:520-539.

Fischbein, N.J., Dillon, W.P., and Barkovich, A.J. 2000. Teaching Atlas of Brain Imaging. pp. 248249, 329-332. Thieme, New York.

Shellock, F.G. and Kanal, E. 1996. Magnetic Resonance Bioeffects, Safety and Patient Management. Lippincott Williams and Wilkins, Philadelphia.

Tsai, F.Y., Wang, A., Matovich, V.B., Lavin, M., Berberian, B., Simonson, T.M., and Yuh, W.T. 1995. MR staging of acute dural sinus thrombosis: Correlation with venous pressure measurements and implications for treatment and prognosis. Am. J. Neuroradiol. 16:1021-1029.

Yuh, W.T., Simonson, T.M., Wang, A.M., Koci, T.M., Tali, E.T., Fisher, D.J., Simon, J.H., Jinkins, J.R., and Tsai, F. 1994. Venous sinus occlusive disease: MR findings. Am. J. Neuroradiol. 15:309-316.

\section{Key References}

Bianchi et al., 1998. See above.

These authors describe the importance of MRI and $M R V$ in the diagnosis and follow-up of cerebral venous thrombosis. They point out how different signals on spin echo $T_{1}$ - and $T_{2}$-weighted images evolve according to hemoglobin degradation causing pitfalls and artifacts that must be recognized.

Fischbein et al., 2000. See above.

These authors afford a current concise look at dural sinus thrombosis. In addition to imaging findings, they discuss, clinical findings, treatment, complications, pearls, and pitfalls.

Shellock and Kanal, 1996. See above.

Covers a number of important patient management issues related to MR imaging, including recommended safety procedures, a list of metallic implants that have been tested for MR compatibility, and a list of other sources on MR safety.

Contributed by F. Allan Midyett, Suresh Mukherji, and Laurie Fisher University of North Carolina at Chapel Hill Chapel Hill, North Carolina 